\title{
Identification of genes with a correlation between copy number and expression in gastric cancer
}

\author{
Lei Cheng ${ }^{1,4 \dagger}$, Ping Wang ${ }^{2 \dagger}$, Sheng Yang ${ }^{1}$, Yanqing Yang ${ }^{1}$, Qing Zhang ${ }^{3}$, Wen Zhang ${ }^{4}$, Huasheng Xiao ${ }^{4}$, \\ Hengjun $\mathrm{GaO}^{4^{*}}$ and Qinghua Zhang ${ }^{1,4^{*}}$
}

\begin{abstract}
Background: To elucidate gene expression associated with copy number changes, we performed a genome-wide copy number and expression microarray analysis of 25 pairs of gastric tissues.

Methods: We applied laser capture microdissection (LCM) to obtain samples for microarray experiments and profiled DNA copy number and gene expression using 244K CGH Microarray and Human Exon 1.0 ST Microarray.

Results: Obviously, gain at $8 q$ was detected at the highest frequency (70\%) and 20q at the second (63\%). We also identified molecular genetic divergences for different TNM-stages or histological subtypes of gastric cancers. Interestingly, the C20orf11 amplification and gain at 20q13.33 almost separated moderately differentiated (MD) gastric cancers from poorly differentiated (PD) type. A set of 163 genes showing the correlations between gene copy number and expression was selected and the identified genes were able to discriminate matched adjacent noncancerous samples from gastric cancer samples in an unsupervised two-way hierarchical clustering. Quantitative RT-PCR analysis for 4 genes (C20orf11, XPO5, PUF60, and PLOD3) of the 163 genes validated the microarray results. Notably, some candidate genes (MCM4 and YWHAZ) and its adjacent genes such as PRKDC, UBE2V2, ANKRD46, ZNF706, and GRHL2, were concordantly deregulated by genomic aberrations.

Conclusions: Taken together, our results reveal diverse chromosomal region alterations for different TNM-stages or histological subtypes of gastric cancers, which is helpful in researching clinicopathological classification, and highlight several interesting genes as potential biomarkers for gastric cancer.
\end{abstract}

Keywords: Copy number variations, Gene expression profile, Correlation, Biomarkers, Gastric cancer

\section{Background}

Despite its steady declining trend worldwide, gastric cancer is still the second most common cause of cancer related deaths with 700,000 cases annually [1]. Due to no symptoms at the early stage of gastric cancer, it is often detected at the advanced stage and the prognosis for treatment at that time is poor [2]. Therapeutic interventions to treat such late stage carcinomas are usually restricted to noncurative gastrectomy, lymphadenectomy and postoperative chemoradiotherapy. Thus, five-year relative survival rates of

\footnotetext{
*Correspondence: hengjun_gao@shbiochip.com; qinghua_zhang@shbiochip. com

${ }^{\dagger}$ Equal contributors

${ }^{1}$ State Key Laboratory of Medical Genomics and Shanghai Institute of Hematology, Ruijin Hospital, Shanghai Jiaotong University School of Medicine, Shanghai, China

${ }^{4}$ National Engineering Center for Biochip at Shanghai, Shanghai, China Full list of author information is available at the end of the article
}

gastric cancer patients barely reach below $30 \%$ in most countries [3]. It is of great clinical importance to identify new biomarkers for early diagnosis, targeted treatment and prognosis evaluation in gastric cancer.

Gastric cancers can be divided into two main histological subtypes, differentiated and poorly differentiated (PD) adenocarcinomas. Differentiated adenocarcinoma is defined by tubular or glandular formation with cancer cells similar to the intestinal metaplasia, whereas the PD type is characterized by disruption of tubular formation due to reduction or loss of cell-cell interaction [4]. The PD adenocarcinomas occur in relatively young individuals and often metastasize to the peritoneum or lymph nodes, resulting in a poor prognosis [5]. Despite the aggressive nature of PD gastric cancer, little is known about the precise mechanisms of carcinogenesis or progression and specific therapeutic targets.

\section{Biomed Central}


It is currently realized that multiple genetic aberrations accumulating during the long process of carcinogenesis are responsible for the initiation and progression of cancers [6]. DNA copy number variations ( $\mathrm{CNVs}$ ) are important influential factors for altered gene expression levels in cancer. Recently, integration of genome-wide array-based comparative genomic hybridization (aCGH) and gene expression microarray data has provided a new insight about the molecular mechanisms underlying gene expression alterations [7-10]. In previous studies, various microarrays (cDNA, BAC or PAC clone, oligo) were applied to investigate CNVs of gastric cancer. Due to the limit of resolution, sample size and preparation method, the impact of CNVs on gene expression remains poorly understood.

In this study, we performed genome-wide DNA copy number and gene expression profiling of 25 pairs of gastric tissues to identify genes that show correlated patterns of variations. Our study applied laser capture microdissection (LCM) to reduce the contamination of cancer cells by non-cancer cells. We also analyzed aberration patterns of different gastric cancer histopathology subtypes to highlight molecular markers with potential clinical significance.

\section{Results}

DNA copy number variations in gastric cancer

The 27 pairs of gastric samples were analyzed by aCGH as shown in Additional file 1: Table S3. The CNVs of all the chromosomes were displayed in Figure 1A. The frequency of CNVs was detected across the entire genome (Figure 1B). Noticeably, chromosomes 8, 20, and 7 contained more genes undergoing frequent copy number amplifications, whereas the high frequent copy number deletions were observed on chromosomes $6,3,4$, and 18. CNVs frequently detected in gastric cancer were summarized in Table 1 . The gained regions detected in at least $25 \%$ of the samples were located at 8 p11-q24,
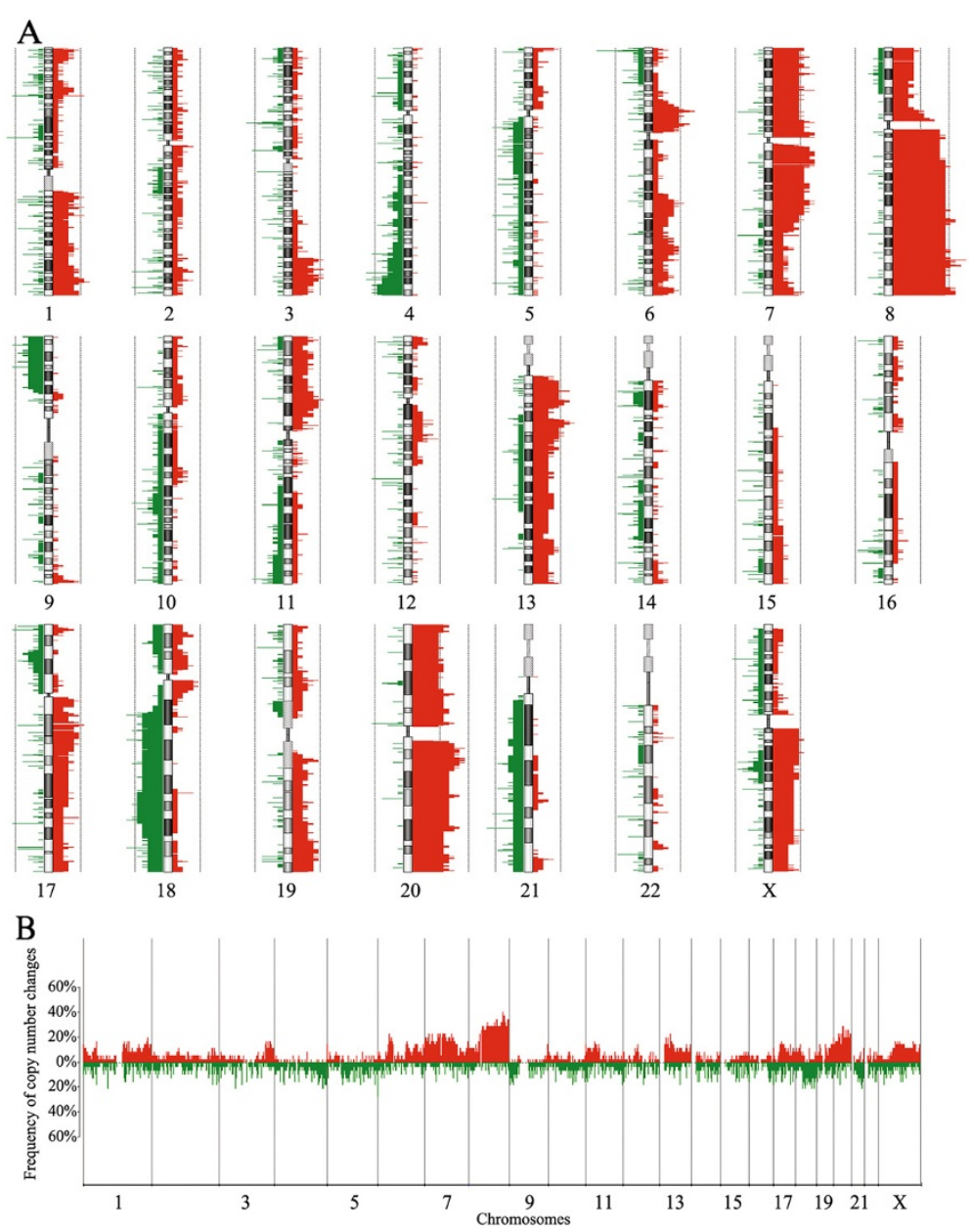

Figure 1 DNA copy number change profiles in the 27 pairs of gastric samples. (A) Summary of chromosomal aberrations was shown. (B) The CNVs frequency of the whole genome was analyzed by aCGH. Gains were marked in red and losses in green. 
Table 1 Chromosomal copy number gains and losses detected in at least $25 \%$ of gastric cancer samples

\begin{tabular}{ll}
\hline Chromosomal aberration & Samples, $\mathbf{n}=\mathbf{2 7}(\%)$ \\
\hline Gains & $19(70 \%)$ \\
\hline $2011-q 24$ & $17(63 \%)$ \\
\hline $7 q 21-q 22$ & $12(44 \%)$ \\
\hline $7 p 12-p 11$ & $11(41 \%)$ \\
\hline $20 p 12-p 11$ & $11(41 \%)$ \\
\hline $7 p 21$ & $10(37 \%)$ \\
\hline $7 q 11$ & $10(37 \%)$ \\
\hline $13 q 13-q 14$ & $9(33 \%)$ \\
\hline $6 p 21$ & $8(30 \%)$ \\
\hline $6 p 12$ & $8(30 \%)$ \\
\hline $7 p 15$ & $8(30 \%)$ \\
\hline $13 q 12$ & $8(30 \%)$ \\
\hline $20 p 13$ & $8(30 \%)$ \\
\hline $1 q 42$ & $7(26 \%)$ \\
\hline $7 p 22$ & $7(26 \%)$ \\
\hline Losses & \\
\hline $4 q 34$ & $9(33 \%)$ \\
\hline $6 p 25$ & $9(33 \%)$ \\
\hline $18 q 12$ & $8(30 \%)$ \\
\hline $18 q 22$ & $7(26 \%)$ \\
\hline $3 p 14$ &
\end{tabular}

20q11-q13, 7q21-q22, 7p12-p11, 20p12-p11, 7p21, 7q11, 13q13-q14, 6p21, 6p12, 7p15, 13q12, 20p13, 1q42, and 7 p22 in decreasing order of frequency (Table 1). Regions of loss detected in at least $25 \%$ of the samples were located at $4 \mathrm{q} 34,6 \mathrm{p} 25,18 \mathrm{q} 12,18 \mathrm{q} 22$, and $3 \mathrm{p} 14$ in decreasing order of frequency (Table 1). Minimal common regions of these copy number aberrations were shown in Table 2, including the size, frequency, possible target genes, and chromosomal position of the alteration in base pairs. Possible target genes were selected with at least two-fold copy number associated changes in gene expression levels.

\section{Gastric cancers of different TNM-stages and histological subtypes show diverse copy number aberrations}

We compared DNA copy number aberration profiles of different gastric cancer TNM-stages and histological subtypes, and found 11 noteworthy regions that displayed differences in copy number changes between PD $(n=10)$ and $\mathrm{MD}(\mathrm{n}=11)$ (Figure 2A). Of these, 2 regions (3p14.1 and $19 \mathrm{p} 13.12)$ were more commonly altered in PD cancers compared with the MD type. Seven regions (1p36.33, 6p24.3, 7p21.1, 7p15.2, 20p12.1, 20q12, and 20q13.2) were more commonly altered in the MD type, and 2 regions (20p11.21 and 20q13.33) were significantly altered in both groups. We observed that chromosome 20 showed more different regions in copy number variations between PD and MD type. Moreover, we found that the MD type could be classified by amplification of C20orf11 at 20q13.33 (two-sample $t$-test, $P<0.05$ ) (Figure 3).

Besides, we also performed the comparisons in copy number changes for T1-2 $(\mathrm{n}=11)$ versus T3-4 $(\mathrm{n}=13)$ as well as N0 $(n=14)$ versus N1-3 $(n=11)$. We found that the frequencies of loss at 4p16.1, 4p14, 4q13.2, 5q21.1, 9q21.13, 9q22.31, 10q22.1, 12q15, 14q24.2, 22q11.21, and 22q12.2 were significantly higher in T3-4 stages than in T1-2 stages, while one region (10q22.1) showed more gains in T1-2 stages. One region, 6q15 had both significant gains and losses in T3-4 stages compared to T1-2 stages (Figure 2B). DNA copy number variation profiling of N0 and N1-3 stages also revealed 18 significantly altered genomic regions (1q32.2, 1q42.12, 2q14.1, 2q14.2, 2q35, 3p25.3, 3q26.2, 5q33.3, 6q16.3, 6q22.33, 7q11.22, 9p23, 9q33.2, 11q23.3, 11q25, $14 \mathrm{q} 11.2,14 \mathrm{q} 32.11$, and $15 \mathrm{q} 24.1$ ) which showed more aberrations in N1-3 stages compared with N0 stage (Figure 2C).

\section{Copy number associated gene expression changes}

We found that 163 individual genes showed at least a 1.3-fold copy number associated alteration in their expression (range $1.3-9.8$, median 1.4) (Additional file 2: Table S4). Of these, there was no gene located in the recurrent regions of copy number loss. The gene showing the highest correlation was PI3 (FC=9.8). PI3 (peptidase inhibitor 3, skin-derived $(S K A L P)$ ) gene, amplified in the 20q12-q13.2 region, displayed the strongest copy number amplification correlated overexpression in gastric cancer. Generally, the highest gene expression fold changes between tumor samples with and without copy number amplifications were detected at the $6 \mathrm{p}$ region since out of the 20 genes showing $>1.7$-fold copy number associated changes in their expression, 11 $(55.0 \%)$ were located in the $6 \mathrm{p}$ region (Additional file 2: Table S4). Altogether those included genes showing significant enrichment (Score $>1.3$ ) in basic functions such as DNA replication (TOP1, MCM4, POLB, and MCM3). An unsupervised two-way (genes and samples) hierarchical clustering of 25 pairs of tissue samples based on these genes revealed two distinct clusters separating matched adjacent noncancerous samples from gastric cancer samples (Additional file 3: Figure S2). We performed qRT-PCR for 4 genes (C20orf11, XPO5, PUF60, and PLOD3) from the 163 genes. These genes showed statistically significant copy number associated gene expression alterations, suggesting that our microarray data are reliable (Figure 4).

To further highlight these 163 genes obtained by gene expression fold change, Pearson correlation coefficients between copy number $\log _{2}$ ratios and expression $\log _{2}$ ratios for 
Table 2 Minimal common regions of recurrent ( $\geq 25 \%$ ) copy number amplification and deletion

\begin{tabular}{|c|c|c|c|c|}
\hline Aberration & Position (Mb) & Size $(\mathrm{Mb})$ & Frequency, $n=27$ (\%) & Possible target genes \\
\hline$+1 \mathrm{q} 42.3$ & $233.85-233.86$ & 0.02 & $7(26 \%)$ & - \\
\hline$+6 \mathrm{p} 21.1$ & $43.41-44.34$ & 0.94 & $8(30 \%)$ & MRPL14, POLR1C, HSP9OAB1, XPO5, MRPS18A \\
\hline$+6 \mathrm{p} 12.2$ & $52.22-52.43$ & 0.20 & $7(26 \%)$ & MCM3 \\
\hline$+6 p 12.2-p 12.1$ & $52.44-52.98$ & 0.54 & $7(26 \%)$ & TMEM14A \\
\hline$+7 p 21.1$ & $16.68-17.28$ & 0.60 & $8(30 \%)$ & $B Z W 2$ \\
\hline$+7 p 12.2$ & $50.62-50.67$ & 0.05 & $7(26 \%)$ & - \\
\hline$+7 p 12.1-p 11.1$ & $53.84-57.47$ & 3.63 & $9(33 \%)$ & MRPS17, CCT6A \\
\hline$+7 q 11.21-q 11.23$ & $63.35-73.62$ & 10.27 & $9(33 \%)$ & CLDN4, EIF4H, SBDS, WBSCR22 \\
\hline$+7 q 11.23$ & 73.63-75.81 & 2.18 & $9(33 \%)$ & $\mathrm{MDH} 2$ \\
\hline$+7 q 21.12-q 21.13$ & $87.54-90.07$ & 2.52 & $7(26 \%)$ & SRI, CLDN12 \\
\hline$+7 q 21.2$ & $91.91-92.53$ & 0.62 & $7(26 \%)$ & - \\
\hline$+7 q 21.3-q 22.1$ & $97.67-100.86$ & 3.19 & $9(33 \%)$ & PLOD3, POP7, ARPC1A, COPS6, BUD31 \\
\hline$+8 p 11.21-q 11.1$ & $42.35-47.79$ & 5.44 & $8(30 \%)$ & VDAC3 \\
\hline$+8 q 11.1-q 24.3$ & $47.86-146.27$ & 98.41 & $19(70 \%)$ & $*$ \\
\hline$+13 q 13.3$ & $39.19-39.28$ & 0.10 & $7(26 \%)$ & - \\
\hline$+13 q 14.11$ & $39.81-40.58$ & 0.77 & $8(30 \%)$ & - \\
\hline$+20 p 13$ & $0.19-0.71$ & 0.52 & $7(26 \%)$ & - \\
\hline+20 p12.1-p11.23 & $17.53-17.98$ & 0.46 & $8(30 \%)$ & - \\
\hline$+20 p 11.21$ & $24.84-25.35$ & 0.51 & $10(37 \%)$ & - \\
\hline$+20 q 11.21-q 12$ & $29.40-40.53$ & 11.12 & $14(52 \%)$ & TPX2, RPN2, POFUT1, CHMP4B, TOP1 \\
\hline$+20 q 12-q 13.2$ & $40.54-52.12$ & 11.57 & $11(41 \%)$ & CTSA, SLPI, MYBL2, PI3, YWHAB, TOMM34, B4GALT5, PIGT, C20orf111 \\
\hline$+20 q 13.33$ & $57.96-62.15$ & 4.19 & $10(37 \%)$ & PSMA7, C20orf11 \\
\hline$-3 p 14.2$ & $60.42-60.48$ & 0.06 & $7(26 \%)$ & - \\
\hline$-6 p 25.3$ & $1.86-2.02$ & 0.16 & $9(33 \%)$ & - \\
\hline$-18 \mathrm{q} 22.1$ & $60.04-60.07$ & 0.02 & $7(26 \%)$ & - \\
\hline
\end{tabular}

each gene were calculated. Out of the 163 genes analyzed, $133(81.6 \%)$ showed statistically significant correlations between DNA copy number and gene expression, with a median correlation coefficient of 0.69 (range 0.40-0.96) (Additional file 2: Table S4). Correlations between copy number change and expression level in two representative genes (XPO5 and MCM4) were exhibited in Additional file 4: Figure S3.

\section{Candidate genes at chromosome $8 \mathbf{q}$}

Obviously, gain at 8q11-q24 was detected at the highest frequency $(70 \%)$ (Table 1). Furthermore, we found that 32 genes selected from genes located at 8q11-q24 via a two-sample $t$-test $(P<0.0001)$ from 50 gastric tissues were overexpression along with copy number gain (excluding GRINA, lack of aCGH data) and revealed a distinct clustering of the genes overexpressed in gastric cancer samples and underexpressed in matched adjacent noncancerous samples (Figure 5).

We noticed that expression of candidate genes located adjacent to MCM4 at $8 \mathrm{q} 11.21$, including
$P R K D C$ and $U B E 2 V 2$, and $Y W H A Z$ at $8 \mathrm{q} 22.3$ including ANKRD46, ZNF706, and GRHL2, showed the same trends as that of MCM4 and YWHAZ, respectively (Figure 6). In addition, these genes were concordantly up-regulated in the samples of gastric cancer with amplification at $8 \mathrm{q} 11.21$ (Figure $6 \mathrm{~A}, \mathrm{c}$ ) or $8 \mathrm{q} 22.3$ (Figure 6B, c).

\section{Discussion}

In this study, we performed a genome-wide analysis of DNA copy number and gene expression changes in gastric cancer to identify genes whose expression are deregulated due to altered copy number and to find potential molecular markers with biological roles in gastric carcinogenesis. Using oligo-based aCGH, gene expression microarrays as well as bioinformatics methods, we acquired genes that were differentially expressed in association with copy number variations. Diverse copy number profiles of different gastric cancer TNM-stages (T1-2 vs. T3-4 and N0 vs. N1-3) and histological subtypes (PD vs. MD) were also shown, implicating the 


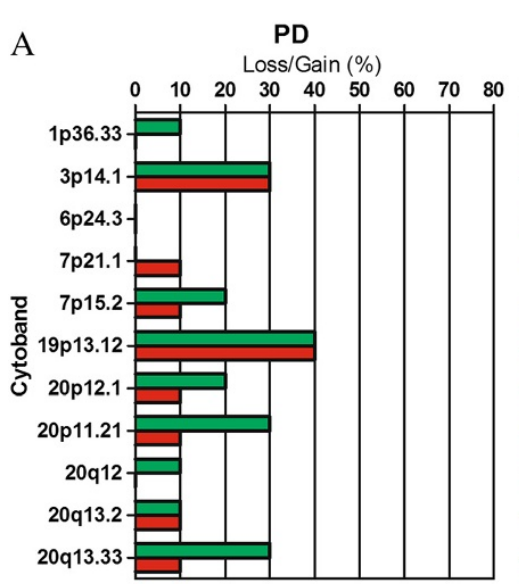

T1-2

B

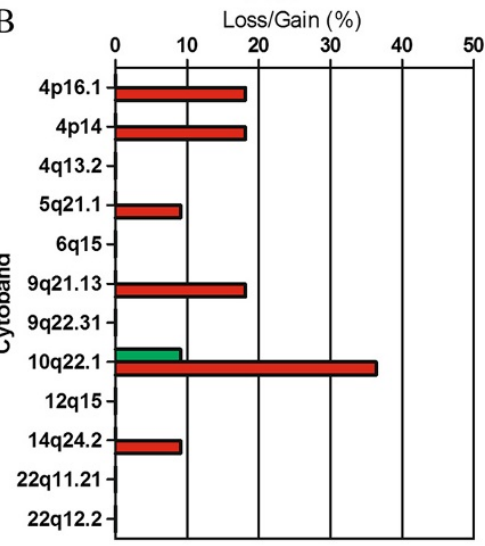

No

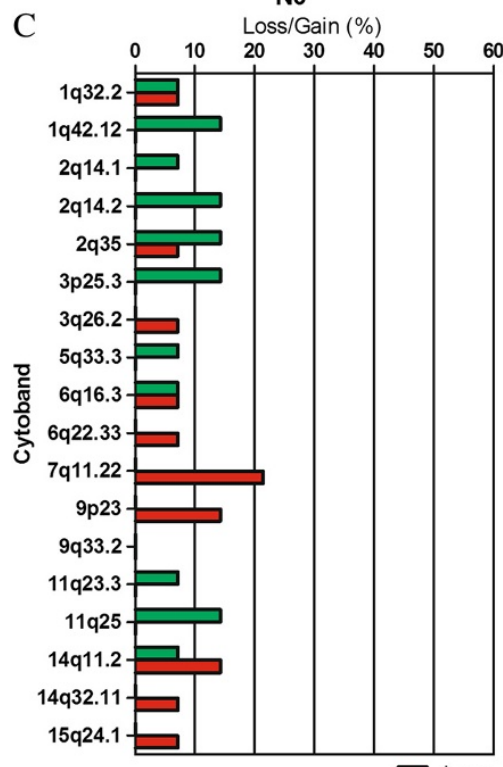

$\square$ Loss

\section{MD}

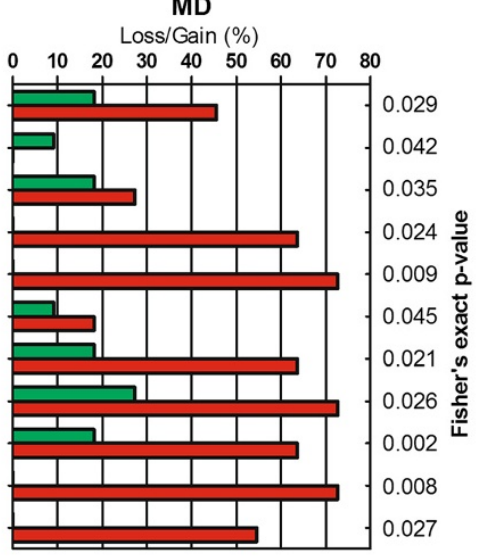

T3-4

Loss/Gain (\%)

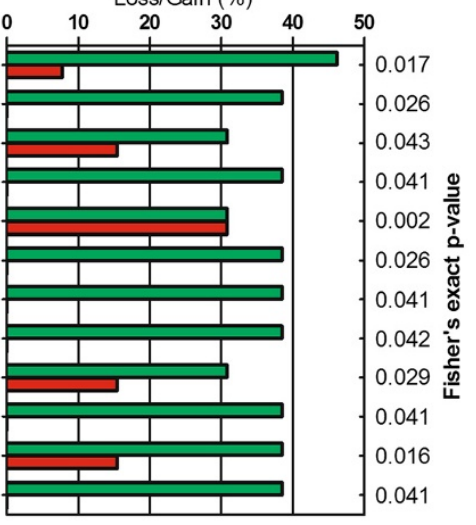

N1-3

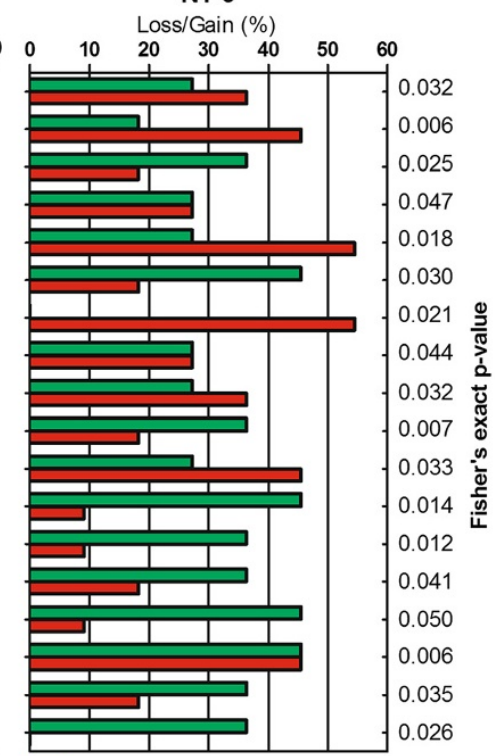

$\square$ Gain

Figure 2 Copy number aberration profiles of different gastric cancer subtypes revealed significantly altered genomic regions. The clinical sample group comparisons were performed for PD $(n=10)$ vs. MD $(n=11)(\mathbf{A})$, T1-2 $(n=11)$ vs. T3-4 $(n=13)(\mathbf{B})$, and N0 ( $n=14)$ vs. N1-3 $(n=11)(C)$. 


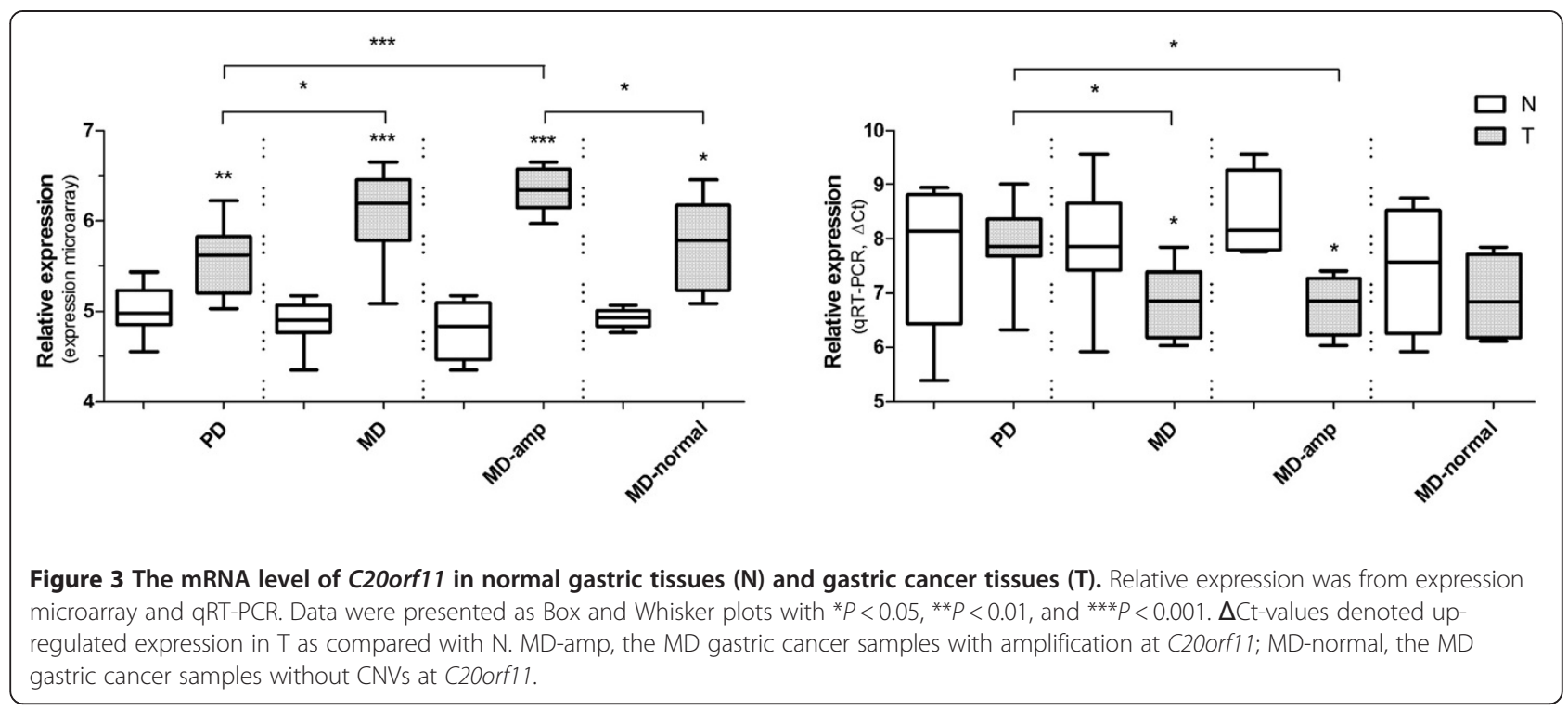

identified copy number regions with valuable biomarkers in diagnostics and in selecting therapy modalities for different gastric cancer subtypes.

On the whole, we identified recurrent copy number gains in 15 chromosomal regions and losses in 5 chromosomal regions which were consistent with the previously published studies [11-20]. Noticeably, gain at 8p11-q24 was detected at the highest frequency (70\%) and 20q11q13 at the second (63\%). Taken together, we speculated that the identified CNVs, especially gain at 8q11-q24 as well as including candidate genes (SULF1, PRKDC, LAPTM4B, GRINA, FAM91A1, GPR172A, PPM2C, MCM4, ENY2, RAD21, SIAHBP1, SLC25A32, PTDSS1, $A T P 6 V 1 C 1$, INTS8, and so on) (Figure 5), may play an important biological role in the pathogenesis of gastric cancer. Indeed, a detailed genomic analysis of chromosome 8q has been performed on gastroesophageal junction (GEJ) adenocarcinomas and this study revealed other genes (ANXA13, MTSS1, FAM84B, C8orf17, and PTK2) except $M Y C$ involved in the 8q amplification and the pathology of GEJ adenocarcinomas [19].

In addition, it was the first time for this findings that expression of MCM4, PRKDC, and UBE2V2 at 8q11.21, or YWHAZ, ANKRD46, ZNF706, and GRHL2 at 8q22.3 was co-regulation and was concordantly up-regulated in the samples of gastric cancer with amplification at $8 \mathrm{q} 11.21$ or $8 \mathrm{q} 22.3$. MCM4 is one of the highly conserved mini-chromosome maintenance proteins (MCM) that are essential for the initiation of eukaryotic genome replication and is highly expressed in esophageal cancer and cervical squamous cell carcinoma [21,22]. Although negative DNA-PKcs (DNA-dependent protein kinase catalytic subunit, also known as PRKDC) expression has been reported to be found in about 20\% (114/564) of human gastric cancers and be associated with gastric cancer progression and poor patient survival, especially for stage I gastric cancer patients [23,24], it is positively expressed in $36.8 \%(82 / 223)$ of nasopharyngeal carcinoma tissues and is in association with low 5 -year overall survival rate [25]. In our study, PRKDC was up-regulated (at least a two-fold change in the gene expression level) in $64 \%(16 / 25)$ of gastric cancer samples. Details of its expression in human cancer are controversial, so further studies will be needed to clarify the mechanism for PRKDC. It has been reported that hMMS2 (methyl methanesulfonate sensitive 2 , S. cerevisiae, homolog of, also known as UBE2V2) serves a redundant role in human PCNA polyubiquitination [26]. Therefore, we speculated that these overexpressed genes located at 8q11.21 may concordantly play an important role in the pathogenesis of gastric cancer. Indeed, a recent study has also shown that genes located adjacent to EGFR at 7p11 or SMAD4 at $18 \mathrm{q} 21$ were in close association with one another and may play a role in the pathogenesis of advanced gastric carcinoma [9]. Although YWHAZ at $8 \mathrm{q} 22.3$ has been considered as a potent antiapoptotic gene [27], we cannot exclude the possibility that other candidate genes may also be present in the region.

Gastric cancers of different TNM-stages or histological subtypes display diverse copy number aberrations. In our study, the MD type tended to be distinguished by gains of C20orf11 at 20q13.33. It has been reported to the higher frequency of $20 \mathrm{q}$ amplifications in intestinal gastric cancer [20]. A study has also previously shown that copy number gains at $20 \mathrm{q}$ are significantly frequent in cell lines derived from tumors of the well-differentiated type [28]. Genetic divergence was also revealed between the T1-2 and T3-4 

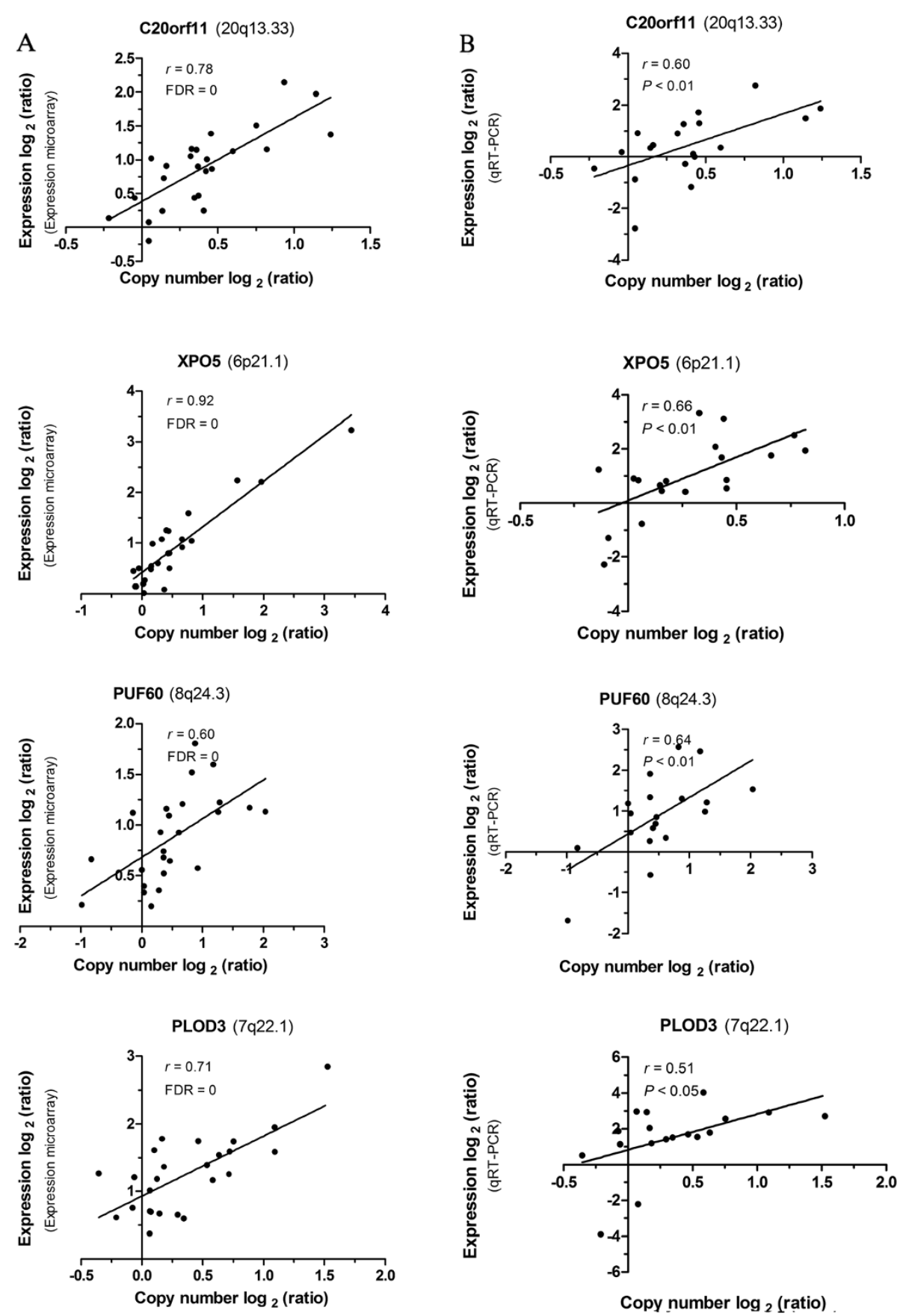

Figure 4 Correlation between copy number ratios and expression ratios in representative genes was validated by qRT-PCR. Each dot indicates an individual sample. The $X$ axis displays copy number log ratios and the $Y$ axis shows gene expression log ratios from microarray (A) or qRT-PCR (B). FDR, false discovery rate.

stages. We found that $4 \mathrm{p} 16.1,4 \mathrm{p} 14,5 \mathrm{q} 21.1,9 \mathrm{q} 21.13$, 10q22.1, and 14q24.2 showed copy number gains in T1-2 and copy number losses in the T3-4 stages. Two regions, 9q22.31 and 22q12.2 both had significant losses in the T34 stages. In addition, 9p23 and 15q24.1 were found to be more common gains in $\mathrm{N} 0$ and losses in N1-3 type gastric cancers. It was the first time to in detail give DNA copy number profiles of different gastric cancer TNM-stages and histological subtypes. Taken together, these studies provided a new insight about researching pathological classification which is helpful to estimate prognosis or personalized therapy for different gastric cancer subtypes. 


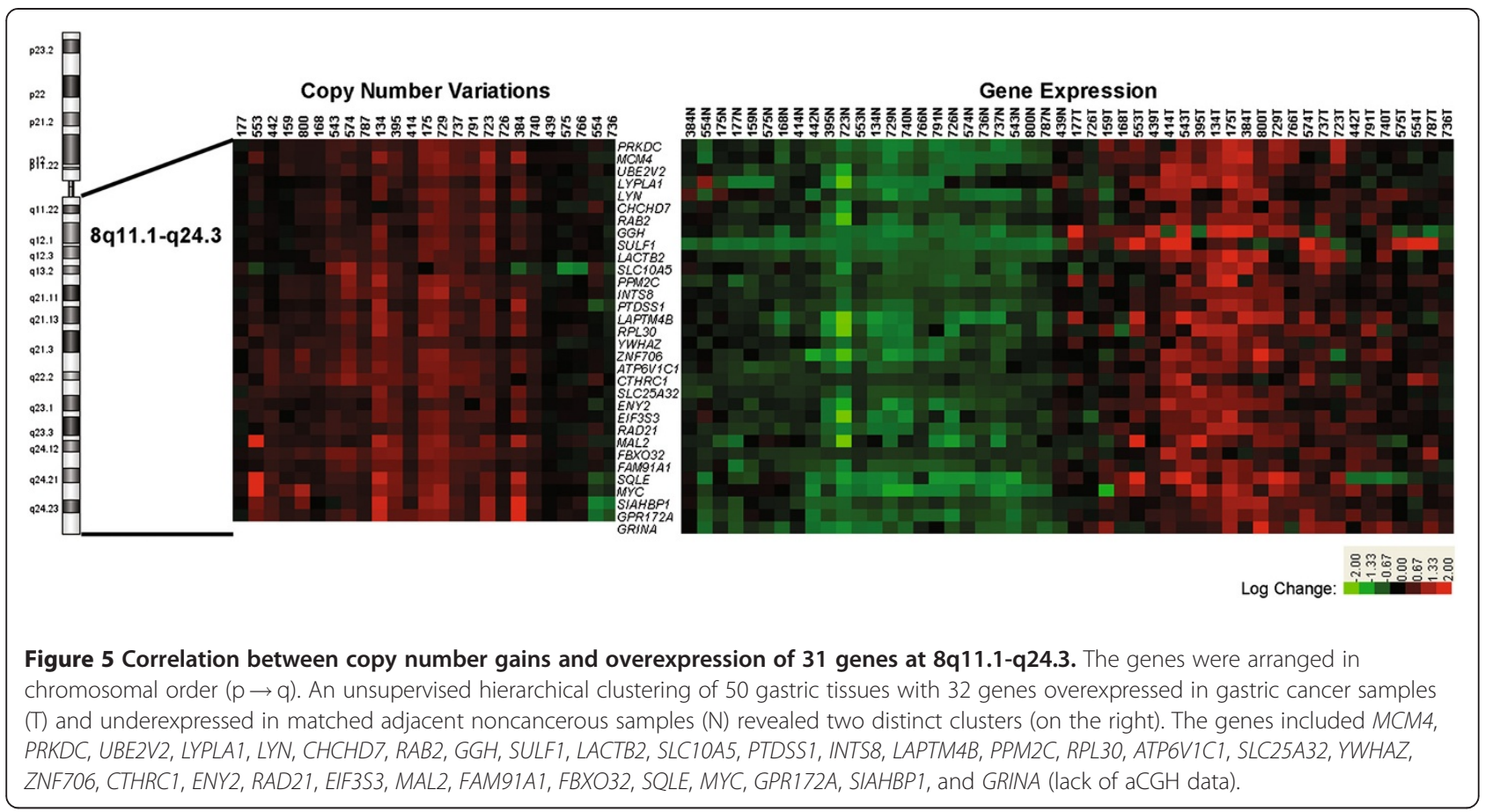

On the other hand, we discovered 163 genes whose expression was deregulated in association with copy number variation. Combining the other recent studies, our study revealed 12 overlapping genes: POLR1C (6p21.1), LANCL2 (7p12.1-p11.1), CCT6A (7p12.1-p11.1), MRPS17 (7p12.1p11.1), SMURF1 (7q21.3-q22.1), COPS6 (7q21.3-q22.1), SQLE (8q11.1-q24.3), RRBP1 (20p12.1-p11.23), SNX5 (20p12.1-p11.23), ID1 (20q11.21-q12), PI3 (20q12-q13.2), and PARD6B (20q12-q13.2) in at least one of the previously published studies [7-10]. Novel genes included SIAHBP1, ATP6V1C1, SLC25A32, ZFAND1, MCM4, XPO5, PLOD3, PSMA7, EIF3S6, TPD52, NSMCE2, $M R P S 18 A, S T K 3$, and MAD2L1BP with no previous gastric cancer associated reports. Moreover, 17 of the identified genes (CLDN4, SRI, MYC, PRKDC, SLPI, LAPTM4B, MYBL2, YWHAB, YWHAZ, MCM3, SERPINE1, SLC29A1, ID1, CDK6, EIF2C2, PTK2, and GSTA1) have previously been implicated in gastric cancer, and six of the genes (MYC, SBDS, CHCHD7, TOP1, COX6C, and $C D K 6)$ are included in the Cancer Gene Census [29].

Based on previous studies [30-32], we applied 1.3-fold cut-off for selecting genes with alteration in their expression. Moreover, we performed Pearson correlation analysis between copy number and expression for these 163 correlated genes to further highlight them. Out of the genes analyzed, 133 (81.6\%) showed statistically
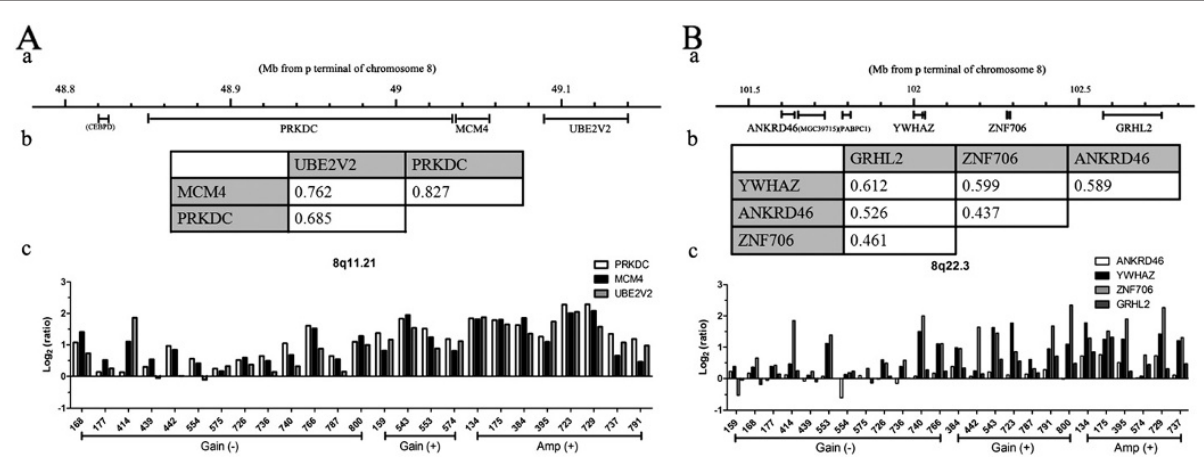

Figure $\mathbf{6}$ Concordant deregulation of candidate genes at $\mathbf{8 q 1 1 . 2 1}$ and $\mathbf{8 q 2 2 . 3}$. Expression of candidate genes located adjacent to MCM4 at $8 q 11.21$ (A) and YWHAZ at 8q22.3 (B) shows the same trends as that of MCM4 and YWHAZ, respectively. (a) Schematic genome structures of neighboring candidate genes at 8q11.21 and 8q22.3; (b) Pearson's correlation coefficients of expression log ratios among candidate genes; (c) Expression log ratio of the individual gene in each sample. Gain (-)/Gain (+) was referred to samples with or without gain at 8q11.21 or 8q22.3; Amp (+) was referred to samples with amplification at $8 \mathrm{q} 11.21$ or $8 \mathrm{q} 22.3$. 
significant correlations between DNA copy number and gene expression (Additional file 2: Table S4). According to gene expression fold changes (FC), PI3 showed the highest correlation $(\mathrm{FC}=9.8)$. But its Pearson correlation coefficient was 0.18 . So the contradiction revealed that the method applying gene expression fold changes[10] to obtain correlated genes was not a strong manner.

To validate the microarray results, four genes (C20orf11, XPO5, PUF60, and PLOD3) were selected for qRT-PCR. The C20orf11 gene displayed copy number correlated overexpression in MD type gastric cancer according to the microarray and qRT-PCR analysis (Figure 3). To our knowledge, no previous report regarding the possible tumor association of C20orf11 has been published. Twa1 (two hybrid-associated protein 1 with RanBPM), also known as C20orf11, was well conserved through evolution and was localized within the nucleus. Interestingly, Twal was found to possess the LisHCTLH motif which is detected in proteins involved in microtubule dynamics, cell migration, nucleokinesis and chromosome segregation [33]. A study indicated that both Twa1 and hMuskelin comprise a protein complex with RanBPM [34]. It has been shown that XPO5 (Exportin-5) is key to miRNA biogenesis and may help coordinate nuclear and cytoplasmic processing steps [35]. Exportin-5 controls Dicer1 expression posttranscriptionally and alterations in miRNA expression can strongly influence cellular physiology [36]. A recent study has shown that the XPO5 genetic defect traps premiRNAs in the nucleus of cancer cells, reduces miRNA processing, and diminishes miRNA-target inhibition. Importantly, the restoration of XPO5 functions reverses the impaired export of pre-miRNAs and has tumorsuppressor features in a subset of cancers with microsatellite instability $\left(\mathrm{MSI}^{+}\right)$[37]. In our study, XPO5 exhibited copy number associated overexpression in gastric cancer. PUF60 (poly-U binding splicing factor 60KDa, also known as FIR and SIAHBP1) has been reported to regulate $c-m y c$ transcription through the general transcription factor TFIIH [38]. A study has displayed that the deficiency of LH3 (lysyl hydroxylase 3, also known as PLOD3) glycosyltransferase activities, especially in the extracellular space, causes growth arrest [39]. Due to the limitation in the number of samples, correlations between copy number alteration and gene expression level from qRT-PCR were almost lower than the microarray data. In all, qRT-PCR analysis validated the microarray results and highlighted some interesting genes as potential target genes.

\section{Conclusions}

In conclusion, the integrated analysis of gene copy number and expression pointed out several interesting genes as potential biomarkers for gastric cancer although further studies need to be performed. We also identified diverse chromosomal regions involved in different TNM-stages or histological subtypes of gastric cancer. Taken together, these results were helpful in clinical stages and early diagnosis or treatment of gastric cancer.

\section{Methods}

\section{Samples and laser capture microdissection}

A total of 27 gastric cancer tissues and matched adjacent noncancerous tissues were obtained from the tissue bank of Shanghai Biochip Center (SBC), which were collected immediately after surgical resection and snap-frozen in liquid nitrogen, then stored in the tissue bank of SBC till later use. Informed consent was obtained from each participating patient. Ethics approval for this study was granted by the Human Research Ethics Committee of Shanghai Jiaotong University School of Medicine. All tissue samples were double examined with hematoxylin $\mathcal{E}$ eosin staining method by two individual pathologists. The clinical and pathological information on the patients was summarized in Additional file 5: Table S1. All tumors were reviewed for invasion $(\mathrm{T})$, lymph node status $(\mathrm{N})$, and metastasis (M). Distant metastasis (M1) was seen in 6 cancer samples. Our cancer tissue samples consisted of two differentiation subtypes: PD $(n=12)$ and moderately differentiated ( $M D, n=11$ ) besides moderately-poorly differentiated $(M-P D, n=4)$ type. All gastric cancer samples were adenocarcinomas and two of the tumor samples showed partial signet-ring cell carcinomas.

Sections of $8 \mu \mathrm{m}$ thickness were produced on a Microm HM 550 microtome (Microm, Walldorf, Germany) and mounted on room-temperature Silane Prep slides (MMI, Glattbrugg, Switzerland). After staining with HistoGene LCM Frozen Section Cresyl Violet Staining Kit (Ambion, Austin, TX), microdissection was performed using MMI CellCut LCM system (MMI), under $100 \times$ magnification. Tumor or non-malignant cells were captured from cancerous or adjacent noncancerous tissues using LCM macro caps (MMI), respectively, and the number of about 5,000 cells was collected within 40 minutes after cryo-section for each slide. Additional file 6: Figure S1 showed efficiency of cell capturing.

\section{Genomic DNA and total RNA preparation}

Following tissue collection, the caps with the captured cells were incubated in $50 \mu \mathrm{l}$ buffer ATL of QIAamp DNA Micro Kit (Qiagen, Hilden, Germany), stored in $4^{\circ} \mathrm{C}$ for later genomic DNA isolation, or incubated in $100 \mu \mathrm{l}$ of lysis solution of RNAqueous-Micro Kit (Ambion) at $42^{\circ} \mathrm{C}$ for 30 minutes, then frozen at $-80^{\circ} \mathrm{C}$ till later total RNA isolation. Genomic DNA (gDNA) and total RNA were extracted using proper reagents according to the manufacturer's protocols. The quality 
of gDNA was verified on 1\% agarose gel electrophoresis, and DNA concentration was measured using Nanodrop ND-1000 spectrophotometer (Nanodrop Technologies, Wilmington, DE). Total RNA integrity, purity and concentration were determined with Agilent's 2100 Bioanalyzer (Agilent Technologies, Palo Alto, CA) (data not shown). Only RNAs with RNA integrity number (RIN) $>7.0$ were applied in later microarray experiments.

\section{Oligo-based aCGH}

Genomic imbalances of 27 pairs of gastric samples were analyzed by aCGH using 244 K CGH Microarrays containing 244,000 probes with $8.9 \mathrm{~KB}$ overall median probe spacing (7.4 KB in Refseq genes) (Agilent Technologies). The gDNA (500 ng) was digested using Alu I and Rsa I restriction enzymes (Promega, Madison, WI), and labeled with either Cy5- or Cy3-dUTP fluorescent dyes for cancerous and adjacent noncancerous samples, respectively, using Agilent Genomic DNA Labeling Kit Plus (Agilent Technologies). Labeled DNA products were purified with Microcon YM-30 filtration devices (Millipore, Bedford, MA), and DNA yield and dye incorporation were determined. Then equal amount of the labeled sample pairs were mixed and hybridized on CGH microarrays by using the SureHyb chambers, for 40 hours at $60^{\circ} \mathrm{C}$. After washing, the microarray slides were scanned immediately using an Agilent microarray scanner, and raw data were extracted using Feature Extraction Software version 9.5.3 at the default CGH parameter settings (Agilent Technologies).

Putative CNV intervals in each sample were identified using Agilent CGH Analytics software ver. 4.0.76. Cy5/ Cy3 ratios were converted into $\log _{2}$-transformed values. Centralization and fuzzy zero corrections were applied to the microarray. The ADM-2 algorithm at threshold 4 was used to identify the CNVs in individual samples and to determine aberration frequencies in gastric cancer samples. In addition, the following aberration filters were employed: minimum number of probes in region $=2$, minimum absolute average $\log _{2}$ ratio for region $=0.5$, maximum number of aberration regions $=10,000$. The $\log _{2}$ ratio of 0.5 corresponds to a 1.4-fold variation in the DNA copy number. Genes in CNVs were annotated by SCAN [40]. Chromosome Y was removed from the analysis. Original copy number data have been submitted to NCBI's Gene Expression Omnibus (GEO) [41] and are accessible through GEO Series accession number [GEO: GSE33428] (http://www.ncbi.nlm. nih.gov/ geo/query/acc.cgi/acc $=$ GSE33428). Minimal common regions of recurrent variations in the 27 samples were analyzed, including the size and chromosomal position of the aberration. An alteration was defined as recurrent, if it was present in at least $25 \%$ of the samples [10].
To compare DNA copy number aberrations in different gastric cancer TNM-stages or histological subtypes, we used a Fisher's exact test based on the $3 \times 2$ table in each region, with the rows representing different gastric cancer histopathology subtypes and the columns representing number of samples with copy number gain, normal copy number or copy number loss in that region [42]. Statistical significance was recognized with $P$ value $<0.05$. Due to the gender differences between the arrays that could cause bias in the analysis, chromosomes $\mathrm{X}$ and $\mathrm{Y}$ were excluded from the calculation. Two tumor samples which showed partial signet-ring cell carcinomas were also removed from the analysis. The clinical sample group comparisons were performed for PD $(\mathrm{n}=10)$ vs. $\mathrm{MD}(\mathrm{n}=11), \mathrm{T} 1-2(\mathrm{n}=11)$ vs. T3-4 $(\mathrm{n}=13)$ and N0 $(\mathrm{n}=14)$ vs. N1-3 $(\mathrm{n}=11)$. To ensure meaningful copy number patterns, at least 25 percent of the samples had to have classifying gains or losses in at least one of the compared classes.

\section{Gene expression microarray}

Twenty-five pairs of gastric samples were used for gene expression profiling. Total RNAs were labeled with Affymetrix GeneChip Whole Transcript Sense Target Labeling and Control Reagents Kit, and hybridized to Human Exon 1.0 ST microarrays (Affymetrix, Santa Clara, CA). In brief, one hundred nanograms of starting total RNAs was used in first round double strand (ds)-cDNA synthesis and cRNA synthesis; 8-10 $\mu \mathrm{g}$ of cRNA could be got and then used in second round single-strand (ss)-cDNA synthesis. Ss-cDNA fragmentation and labeling were finished according to the Whole Transcript Sense Target Labeling Assay manual (Affymetrix). Five micrograms of biotin labeled ss-cDNA was used to hybridize the Human Exon 1.0 ST microarray for 16 hours. Staining and washing were all processed with Affymetrix's protocols. The arrays were scanned on the GeneChip Scanner 30007 G with GeneChip Operating Software ver. 1.3 (Affymetrix) to generate .CEL intensity files.

Expression Consol software (v 1.0) (Affymetrix) integrated robust multiarray analysis (RMA) algorithm was applied to extract gene-level expression signal and detection above background (DABG) $P$-value for each probe set of the samples. Probe sets with $P<0.05$ were considered as present, and an expression signal cutoff was set as 3.9 , as minimum number of falsely called probes. Only the genes with signal above cutoff were used in later analysis. All gene expression data are available at NCBI via GEO [GEO: GSE33335] (http://www.ncbi.nlm. nih.gov/geo/query/acc.cgi/acc $=$ GSE33335).

\section{Integrative analysis of the CGH and expression data}

To investigate the correlation between DNA copy number and gene expression, we only analyzed genes located 
in the chromosomal regions with recurrent aberrations. The purpose of this way was to pinpoint gene expression changes that were associated with alterations in DNA copy number, and could therefore enlighten some potential oncogenes, tumor-suppressor genes and stability genes with functional roles in cancers. Amplifications and deletions were treated separately in the analysis. The median expression level of each gene was compared between cancer samples with and without copy number amplifications/deletions to assess the effect of copy number changes on gene expression. Gene expression fold changes (FC) were calculated by dividing the median expression of the cancer samples with CNVs by the median expression of the cancer samples without copy number alterations [10]. At least 1.3-fold copy number associated aberration in gene expression was selected. The functional annotation analysis of selected genes was performed using the DAVID Database [43,44]. A score of over 1.3 was considered to be a significant level of enrichment in the gene set with a minimum gene count threshold of $\geq 2$. The selection of the genes that displayed the correlations between copy number and expression changes would be expected to exhibit a differential gene expression pattern between normal gastric tissues and gastric tumor tissues [7]. That expectation was realized by hierarchical clustering of 25 pairs of gastric samples with these genes using the average linkage method. Visualization was performed in Java Treeview 1.1.3 software. Pearson correlation coefficients between DNA copy number aberrations and alterations in mRNA expression level for each selected gene were also calculated in SPSS 11.5 software to further highlight these genes with association between copy number and expression. The results from the integrated microarray analysis were compared with four previously published studies that systematically integrated genome-wide gene copy number and expression data [7-10].

In addition, we also analyzed genes located in diverse chromosomal regions of different gastric cancer TNMstages or histological subtypes and identified genes with the correlation between gene copy number and expression change based on the above method.

\section{Quantitative reverse transcription-polymerase chain reaction (qRT-PCR)}

For validation of the microarray data, the qRT-PCR was performed with SYBR Green assay in ABI 7300 Real Time PCR System according to the manufacturer's instructions. All primers (Additional file 7: Table S2) were designed using Primer Express 3 (Applied Biosystems, Foster City, CA). Specificity of primer sets was checked with BLAST. Forward and reverse primers were mixed and diluted to $5 \mu \mathrm{M}$. Two micrograms of total RNAs extracted from 19 pairs of gastric samples was reverse transcribed into cDNA using First Strand cDNA Synthesis Kits (Fermentas, Glen Burnie, Maryland) as was suggested by the manufacturer. Then cDNA was diluted 1:10. The RT-PCR master mixtures consisted of: $1 \mu \mathrm{l}$ primers, $1 \mu \mathrm{l}$ diluted cDNA, $10 \mu \mathrm{l}$ $2 \times$ SYBR Green master mix (TOYOBO, Osaka, Japan), $8 \mu \mathrm{l}$ RNA-free water in a final volume of $20 \mu \mathrm{l}$. All assays were normalized by the ACTB internal control. Thermal cycling conditions comprised $50^{\circ} \mathrm{C}$ for $2 \mathrm{~min}, 95^{\circ} \mathrm{C}$ for $5 \mathrm{~min}$, followed by 40 cycles of $95^{\circ} \mathrm{C}$ for $15 \mathrm{~s}$ and $60^{\circ} \mathrm{C}$ for $1 \mathrm{~min}$. All reactions were performed in triplicate. Relative quantification results were analyzed using the $2^{-\Delta \Delta C t}$ method.

\section{Additional files}

\begin{abstract}
Additional file 1: Table S3 The 27 pairs of gastric samples were analyzed by aCGH using Agilent CGH Analytics 4.0.76 software. ADM-2 algorithm with a threshold level of 4 was used to identify CNVs in individual samples. CNVs, copy number variations.

Additional file 2: Table S4 Copy number associated gene expression changes. Pearson correlation coefficients between DNA copy number aberrations and alterations in mRNA expression level for each selected gene were calculated in SPSS 11.5 software. Gene expression referred to $\log _{2}$ ratios from gene expression profiling. Normal and Tumor referred to an average $\log _{2}$ ratio of 25 pairs of gastric samples, respectively. aCGH $\log _{2}$ ratio referred to an average $\log _{2}$ ratio for only those cases (Frequency) in which the ratio was over 1.5-fold changed $\left(\log _{2}\right.$ ratio $\geq 0.585$ or $\leq-0.585$ ). firstly, a mean $\log _{2}$ copy number variation ratio was calculated for all the probes targeting the same gene. Then, the Pearson's $r$ was measured between aCGH and gene expression profiling performed in 25 pairs of gastric samples.

Additional file 3: Figure S2 An unsupervised hierarchical clustering of 50 gastric samples with 163 genes revealed two distinct clusters. Log ratio scale bar for the Treeview color change was also shown. Suffix " $T$ " indicates gastric cancer samples; "N" indicates matched adjacent noncancerous samples.
\end{abstract}

Additional file 4: Figure S3 Correlation between copy number ratios and expression ratios in representative genes (XPO5 and MCM4). The X axis showed 25 gastric samples and the $Y$ axis displayed log ratios of copy number and gene expression from microarrays.

Additional file 5: Table S1 Clinical and histological data of the 27 pairs of gastric samples. M, male; F, female; ADC, adenocarcinoma; SRCC, signet-ring cell carcinoma; $T$, invasion activity; $N$, lymph node colonization; M, metastasis; Dif, differentiation; Hp, helicobacter pylori; $M D$, moderately differentiated; PD, poorly differentiated; $M-P D$, moderately-poorly differentiated; NA, not available.

Additional file 6: Figure S1 Efficiency of cell capturing. Noncancerous mucosa (A) before and (B) after dissection of the epithelia. (C) Image of the epithelium on the cap. Tumor cells in muscle layer (D) before and (E) after dissection of the tumor cells. (F) Image of the tumor cell on the cap.

Additional file 7: Table S2 All primers were used in the qRT-PCR validation of gene expression microarray data.

\section{Competing interests}

The authors declare that they have no competing interests.

\section{Authors' contributions}

PW and QZ provided gastric samples and helped to draft the manuscript. $\mathrm{Hj}$ G carried out histopathology examination, participated in study design and helped to draft the manuscript. SY, Yq Y, WZ and Hs X carried out array experiments and participated in acquisition of data. LC performed array data analysis and the statistical analysis, carried out qRT-PCR experiments and drafted the manuscript. Qh Z conceived of the study, participated in its 
design and coordination, and helped to draft the manuscript. All authors read and approved the final manuscript.

\section{Acknowledgements}

This research got supports by the National Basic Research Program of China, 973 Program, 2006CB910402, 2012CB22308. The authors give thanks to Junsong Han, Xiaona Zhang, Libo Lin in SBC for their helps in microarray experiment performs, and Zhidong Zhu and Yi Zhang in data analysis.

\section{Author details}

${ }^{1}$ State Key Laboratory of Medical Genomics and Shanghai Institute of Hematology, Ruijin Hospital, Shanghai Jiaotong University School of Medicine, Shanghai, China. ${ }^{2}$ Department of Pathology, Ninth People's Hospital, Shanghai Jiaotong University School of Medicine, Shanghai, China. ${ }^{3}$ Department of Oncology, Gongli Hospital, Shanghai, China. ${ }^{4}$ National Engineering Center for Biochip at Shanghai, Shanghai, China.

Received: 10 December 2011 Accepted: 27 March 2012 Published: 4 May 2012

\section{References}

1. Parkin DM, Bray F, Ferlay J, Pisani P: Global cancer statistics, 2002. CA Cancer J Clin 2005, 55(2):74-108.

2. Powell SM: Stomach cancer. In In the Genetic Basis of Human Cancer. 2nd edition. Edited by Vogelstein B, Kinzler K. New York: McGraw-Hill; 2002:703708.

3. Brenner $H$, Rothenbacher D, Arndt V: Epidemiology of stomach cancer. Methods Mol Biol 2009, 472:467-477.

4. Tahara E: Oncogenes in human gastric carcinoma. Gan To Kagaku Ryoho 1989, 16(6):2149-2155.

5. Jinawath N, Furukawa Y, Hasegawa S, Li M, Tsunoda T, Satoh S, Yamaguchi $T$, Imamura $H$, Inoue $M$, Shiozaki $H$, et al: Comparison of gene-expression profiles between diffuse- and intestinal-type gastric cancers using a genome-wide cDNA microarray. Oncogene 2004, 23(40):6830-6844.

6. Vogelstein B, Kinzler K: Cancer genes and the pathways they control. Nature medicine 2004, 10(8):789-799.

7. Yang S, Jeung HC, Jeong HJ, Choi YH, Kim JE, Jung JJ, Rha SY, Yang WI, Chung HC: Identification of genes with correlated patterns of variations in DNA copy number and gene expression level in gastric cancer. Genomics 2007, 89(4):451-459.

8. Myllykangas S, Junnila S, Kokkola A, Autio R, Scheinin I, Kiviluoto T, Karjalainen-Lindsberg ML, Hollmen J, Knuutila S, Puolakkainen P, et al: Integrated gene copy number and expression microarray analysis of gastric cancer highlights potential target genes. Int J Cancer 2008, 123 (4):817-825.

9. Tsukamoto $Y$, Uchida T, Karnan S, Noguchi T, Nguyen LT, Tanigawa M, Takeuchi I, Matsuura K, Hijiya N, Nakada C, et al: Genome-wide analysis of DNA copy number alterations and gene expression in gastric cancer. $J$ Pathol 2008, 216(4):471-482.

10. Junnila S, Kokkola A, Karjalainen-Lindsberg ML, Puolakkainen P, Monni O: Genome-wide gene copy number and expression analysis of primary gastric tumors and gastric cancer cell lines. BMC Cancer 2010, 10:73.

11. Morohara K, Nakao K, Tajima Y, Nishino N, Yamazaki K, Kaetsu T, Suzuki S, Tsunoda A, Kawamura M, Aida T, et al: Analysis by comparative genomic hybridization of gastric cancer with peritoneal dissemination and/or positive peritoneal cytology. Cancer Genet Cytogenet 2005, 161(1):57-62.

12. van Dekken H, Geelen E, Dinjens WN, Wijnhoven BP, Tilanus HW, Tanke HJ, Rosenberg C: Comparative genomic hybridization of cancer of the gastroesophageal junction: deletion of 14Q31-32.1 discriminates between esophageal (Barrett's) and gastric cardia adenocarcinomas. Cancer Res 1999, 59(3):748-752.

13. Gorringe KL, Boussioutas A, Bowtell DD: Novel regions of chromosomal amplification at $6 \mathrm{p} 21,5 \mathrm{p} 13$, and 12q14 in gastric cancer identified by array comparative genomic hybridization. Genes Chromosomes Cancer 2005, 42(3):247-259.

14. Wu CW, Chen GD, Fann CS, Lee AF, Chi CW, Liu JM, Weier U, Chen JY: Clinical implications of chromosomal abnormalities in gastric adenocarcinomas. Genes Chromosomes Cancer 2002, 35(3):219-231.

15. Nakanishi M, Sakakura C, Fujita Y, Yasuoka R, Aragane H, Koide K, Hagiwara A, Yamaguchi T, Nakamura Y, Abe T, et al: Genomic alterations in primary gastric cancers analyzed by comparative genomic hybridization and clinicopathological factors. Hepatogastroenterology 2000, 47(33):658-662.

16. Sakakura C, Mori T, Sakabe T, Ariyama Y, Shinomiya T, Date K, Hagiwara A, Yamaguchi T, Takahashi T, Nakamura Y, et al: Gains, losses, and amplifications of genomic materials in primary gastric cancers analyzed by comparative genomic hybridization. Genes Chromosomes Cancer 1999 24(4):299-305

17. Weiss MM, Kuipers EJ, Postma C, Snijders AM, Pinkel D, Meuwissen SG, Albertson D, Meijer GA: Genomic alterations in primary gastric adenocarcinomas correlate with clinicopathological characteristics and survival. Cell Oncol 2004, 26(5-6):307-317.

18. Isinger-Ekstrand A, Johansson J, Ohlsson M, Francis P, Staaf J, Jonsson M, Borg A, Nilbert M: Genetic profiles of gastroesophageal cancer: combined analysis using expression array and tiling array-comparative genomic hybridization. Cancer Genet Cytogenet 2010, 200(2):120-126.

19. van Duin M, van Marion R, Vissers KJ, Hop WC, Dinjens WN, Tilanus HW, Siersema PD, van Dekken $\mathrm{H}$ : High-resolution array comparative genomic hybridization of chromosome 8q: evaluation of putative progression markers for gastroesophageal junction adenocarcinomas. Cytogenet Genome Res 2007, 118(2-4):130-137.

20. Vauhkonen H, Vauhkonen M, Sajantila A, Sipponen P, Knuutila S: DNA copy number aberrations in intestinal-type gastric cancer revealed by arraybased comparative genomic hybridization. Cancer Genet Cytogenet 2006, 167(2):150-154.

21. Gan N, Du Y, Zhang W, Zhou J: Increase of Mcm3 and Mcm4 expression in cervical squamous cell carcinomas. Eur J Gynaecol Oncol 2010, 31 (3):291-294.

22. Huang XP, Rong TH, Wu QL, Fu JH, Yang H, Zhao JM, Fang Y: MCM4 expression in esophageal cancer from southern China and its clinical significance. J Cancer Res Clin Oncol 2005, 131(10):677-682.

23. Lee HS, Yang HK, Kim WH, Choe G: Loss of DNA-dependent protein kinase catalytic subunit (DNA-PKcs) expression in gastric cancers. Cancer Res Treat 2005, 37(2):98-102.

24. Lee HS, Choe G, Park KU, do Park J, Yang HK, Lee BL, Kim WH: Altered expression of DNA-dependent protein kinase catalytic subunit (DNAPKCs) during gastric carcinogenesis and its clinical implications on gastric cancer. Int J Oncol 2007, 31(4):859-866.

25. Yan SS, Liu L, Liu ZG, Zeng MS, Song LB, Xia YF: Expression and clinical significance of DNA-PKcs in nasopharyngeal carcinoma. Ai Zheng 2008, 27(9):979-983.

26. Brun J, Chiu R, Lockhart K, Xiao W, Wouters BG, Gray DA: hMMS2 serves a redundant role in human PCNA polyubiquitination. BMC Mol Biol 2008, 9:24.

27. Tsukamoto $Y$, Nakada C, Noguchi T, Tanigawa M, Nguyen LT, Uchida T, Hijiya N, Matsuura K, Fujioka T, Seto M, et al: MicroRNA-375 is downregulated in gastric carcinomas and regulates cell survival by targeting PDK1 and 14-3-3zeta. Cancer Res 2010, 70(6):2339-2349.

28. Takada H, Imoto I, Tsuda H, Sonoda I, Ichikura T, Mochizuki H, Okanoue T, Inazawa J: Screening of DNA copy-number aberrations in gastric cancer cell lines by array-based comparative genomic hybridization. Cancer Sci 2005, 96(2):100-110.

29. Futreal PA, Coin L, Marshall M, Down T, Hubbard T, Wooster R, Rahman N, Stratton MR: A census of human cancer genes. Nat Rev Cancer 2004, 4 (3):177-183.

30. Caceres M, Lachuer J, Zapala MA, Redmond JC, Kudo L, Geschwind DH, Lockhart DJ, Preuss TM, Barlow C: Elevated gene expression levels distinguish human from non-human primate brains. Proc Natl Acad Sci U S A 2003, 100(22):13030-13035.

31. Iwamoto K, Kakiuchi C, Bundo M, Ikeda K, Kato T: Molecular characterization of bipolar disorder by comparing gene expression profiles of postmortem brains of major mental disorders. Mol Psychiatry 2004, 9(4):406-416.

32. Palanker L, Tennessen JM, Lam G, Thummel CS: Drosophila HNF4 regulates lipid mobilization and beta-oxidation. Cell Metab 2009, 9(3):228-239.

33. Emes $R D$, Ponting CP: A new sequence motif linking lissencephaly, Treacher Collins and oral-facial-digital type 1 syndromes, microtubule dynamics and cell migration. Hum Mol Genet 2001, 10(24):2813-2820.

34. Umeda M, Nishitani H, Nishimoto T: A novel nuclear protein, Twa1, and Muskelin comprise a complex with RanBPM. Gene 2003, 303:47-54.

35. Lund E, Guttinger S, Calado A, Dahlberg JE, Kutay U: Nuclear export of microRNA precursors. Science 2004, 303(5654):95-98. 
36. Bennasser Y, Chable-Bessia C, Triboulet R, Gibbings D, Gwizdek C, Dargemont C, Kremer EJ, Voinnet O, Benkirane M: Competition for XPO5 binding between Dicer mRNA, pre-miRNA and viral RNA regulates human Dicer levels. Nat Struct Mol Biol 2011, 18(3):323-327.

37. Melo SA, Moutinho C, Ropero S, Calin GA, Rossi S, Spizzo R, Fernandez AF, Davalos V, Villanueva A, Montoya G, et al: A genetic defect in exportin-5 traps precursor microRNAs in the nucleus of cancer cells. Cancer Cell 2010, 18(4):303-315.

38. Liu J, Kouzine F, Nie Z, Chung HJ, Elisha-Feil Z, Weber A, Zhao K, Levens D: The FUSE/FBP/FIR/TFIIH system is a molecular machine programming a pulse of c-myc expression. EMBO J 2006, 25(10):2119-2130.

39. Wang C, Kovanen V, Raudasoja P, Eskelinen S, Pospiech H, Myllyla R: The glycosyltransferase activities of lysyl hydroxylase 3 (LH3) in the extracellular space are important for cell growth and viability. J Cell Mol Med 2009, 13(3):508-521.

40. Gamazon ER, Zhang W, Konkashbaev A, Duan S, Kistner EO, Nicolae DL, Dolan ME, Cox NJ: SCAN: SNP and copy number annotation. Bioinformatics 2009, 26(2):259-262.

41. Edgar R, Domrachev M, Lash AE: Gene Expression Omnibus: NCBI gene expression and hybridization array data repository. Nucleic Acids Res 2002, 30(1):207-210.

42. Rose AE, Satagopan JM, Oddoux C, Zhou Q, Xu R, Olshen AB, Yu JZ, Dash A, Jean-Gilles J, Reuter $V$, et al: Copy number and gene expression differences between African American and Caucasian American prostate cancer. J Transl Med 2010, 8:70

43. da Huang W, Sherman BT, Lempicki RA: Systematic and integrative analysis of large gene lists using DAVID bioinformatics resources. Nat Protoc 2009, 4(1):44-57.

44. da Huang W, Sherman BT, Lempicki RA: Bioinformatics enrichment tools: paths toward the comprehensive functional analysis of large gene lists. Nucleic Acids Res 2009, 37(1):1-13.

doi:10.1186/1755-8794-5-14

Cite this article as: Cheng et al:: Identification of genes with a

correlation between copy number and expression in gastric cancer $B M C$ Medical Genomics 2012 5:14.

\section{Submit your next manuscript to BioMed Central and take full advantage of:}

- Convenient online submission

- Thorough peer review

- No space constraints or color figure charges

- Immediate publication on acceptance

- Inclusion in PubMed, CAS, Scopus and Google Scholar

- Research which is freely available for redistribution 